\title{
Estimation des propriétés mécaniques à long terme des sédiments de Dunkerque traités à la chaux ou au ciment
}

\section{N.E. ABRIAK ${ }^{1}$ \\ D. WANG 1,2 \\ R. ZENTAR ${ }^{1}$}

${ }^{1}$ Département Génie Givil et Environnemental École des mines de Douai 941, rue Charles Bourseul BP 10838

59508 Douai Cedex, France

${ }^{2}$ Geotechnical Research Institute Hohai University 1 Xikang Road 210098 Nanjing, China
Le travail présenté dans cet article a pour objectif de combler le manque d'information concernant l'évolution des propriétés mécaniques à long terme de sédiments traités à la chaux et/ou au ciment. Les essais de compression et de traction indirecte sont effectués afin de déterminer les résistances des sédiments à différentes échéances et en particulier à 360 jours. La résistance à la traction et le module mesurés à cette dernière échéance permet d'évaluer le potentiel des matériaux pour une utilisation en couche d'assise de chaussée. Les rapports des résistances $R_{v} / R_{t 360}$ et des modules $E_{t} / E_{t 360 j}$ sont calculés expérimentalement puis comparés à ceux préconisés par les normes françaises NF P 98-113 (1994) et NF P 98 114-3 (2001) pour des matériaux standards. A l'exception des essais sur SD6L, les valeurs obtenues sont légèrement en dessous des valeurs proposées pour l'estimation des résistances en traction des matériaux à 360 jours toutefois systématiquement au-dessus des valeurs proposées pour l'estimation des modules d'elasticité à 360 jours.

Mots-clés : sédiments marins, ciment/chaux, propriété à long terme, résistance, classification.

\section{Estimation of long-term mechanical properties of Dunkerque sediments treated with lime or cement}

The present work in this paper aims to make up the lack of information concerning the evolution of long-term mechanical properties of sediments treated with lime and/or cement. The unconfined compression tests and indirect tensile tests are performed to determine the compressive strength and tensile strength of sediments at different curing times and in particular at 360 days. The tensile strength and elastic modulus measured at 360 days allows evaluating the potential use of designed materials in base course layer. The strength ratios $R_{t d} / R_{1360 d}$ and modulus ratios $\mathrm{E}_{\mathrm{tg}} / \mathrm{E}_{1360 \mathrm{~d}}$ are experimentally calculated and then compared to those recommended by French standard NF P 98-113 (1994) and NF P 98 114-3 (2001) for standard materials. With the exception of SD6L, the obtained values are slightly below the proposed values for estimating the tensile strengths of materials at 360 days but always above the proposed values for estimating elastic modules at 360 days.

Key words: marine sediments, cement/lime, long-term property, strength, classification. 


\section{Introduction}

Les sédiments marins sont des matériaux issus de l'érosion de roches mères, ces derniers peuvent être transportés par les cours d'eau ou le vent puis déposés en mer. Ce dépôt de matière dans des emplacements exploités par le trafic maritime peut nécessiter des opérations d'entretien pour retrouver des conditions optimales pour la circulation de navires. En France, la quantité des sédiments de dragage est considérable et potentiellement problématique. Pour les ports maritimes et d'estuaires français, les dragages d'entretien ou d'approfondissement de chenaux génèrent un volume annuel considérable qui oscille entre 50 et $60 \mathrm{Mm}^{3}$ (Levacher et al., 2006). Les sept grands ports maritimes en France comptabilisent à eux seuls 29,2 $\mathrm{Mm}^{3}$, soit près de $80 \%$ du total (CETMEF, 2010). Les solutions traditionnelles comme le clapage en mer et la mise en dépôts à terre ne s'appliquent plus dans le cas où il s'agit de sédiments fins plus ou moins contaminés. La gestion durable de ces sédiments de dragage demeure donc un enjeu important actuellement.

Avec l'augmentation de la demande de matériaux granulaires dans divers domaines parmi lesquels les travaux publics (Jones et al., 2001 ; Hamer et Karius, 2002 ; Scordia et al., 2008 ; Zentar et al., 2008 ; Tran, 2009; Wang et al., 2012), l'utilisation des sédiments marins comme une nouvelle ressource peut dénouer d'une part le problème de gestion des sédiments de dragage et d'autre part l'approvisionnement de secteur avare de matériaux granulaires. Après le prétraitement des sédiments pour enlever de l'eau et éliminer et/ou stabiliser des polluants (matières organiques, métaux. lourds), les grands volumes de sédiments marins représentent une source de matières premières très intéressante, notamment pour un secteur consommateur de granulats tels que la route.

En France, plus de 376 millions de tonnes de matériaux granulaires sont consommés annuellement dans le domaine du génie civil. La moitié de cette quantité est utilisée dans les travaux routiers (UNPG, 2009). Pour l'utilisation des sédiments de dragage en technique routière, beaucoup d'auteurs ont entrepris une série d'études sur les sédiments traités aux liants à l'aide d'essais de laboratoire et d'essai in situ (Scordia et al., 2008 ; Silitonga et al., 2008 ; Zentar et al., 2008 ; Zhu et Liu, 2008 ; Sannier et al., 2009 ; Wang et al., 2012). Dans la plupart de ces études, les essais mécaniques au laboratoire ou in situ sont réalisés pour des échéances inférieures à 90 jours et très peu de résultats sont disponibles pour des échéances au-delà et surtout à 360 jours. De ce fait, les propriétés mécaniques à 360 jours des sédiments traités au ciment ou à la chaux seront principalement étudiées dans la présente étude afin de combler cette lacune.

Les objectifs de la présente étude sont ainsi : 1) d'identifier les caractéristiques physiques des sédiments bruts ; 2) d'étudier les résistances en compression et en traction à 360 jours des sédiments traités aux liants ; 3) d'explorer l'évolution des résistances mécaniques de ces matériaux dans le temps ; 4) de comparer les rapports de résistances $R_{\mathrm{tj}} / \mathrm{R}_{\mathrm{t} 360 \mathrm{j}}$ et de modules $E_{\mathrm{tj}} /$ $E_{t 360 j}$ avec les valeurs définies par la norme NF P 98-113 (1994).

\section{Matériaux et méthodes}

\section{2. \\ Matériaux}

Les sédiments marins utilisés dans ce travail sont issus du port autonome de Dunkerque, premier port régional et troisième port français, et plus particulièrement de sa partie Est. Les sédiments dragués sont liquides de couleur noirâtre et d'odeur nauséabonde en raison de la présence probable de matières organiques. Les matières organiques sont partiellement éliminées par le prétraitement des sédiments humides : infiltration et séchage. Le site de prélèvement des sédiments du port Est de Dunkerque en France est présenté dans la figure 1.

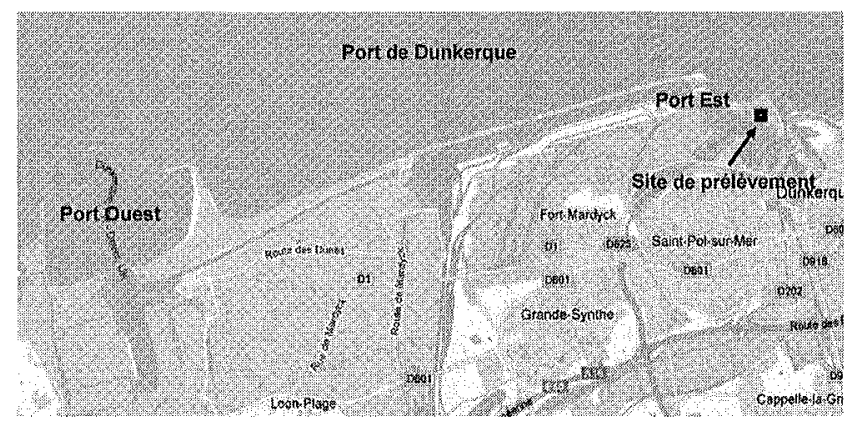

HE. 1 Site de prélèvement des sédiments du port de Dunkerque.

Sampling site of sediments from Dunkirk Port.

Pour le traitement de ces matériaux au liant hydraulique, le ciment utilisé est de type CEM I 42,5 R HSR LA (connu sous le nom de CIBELCOR), ce dernier est obtenu par broyage simultané d'un minimum de $95 \%$ de clinker Portland et d'une faible quantité de sulfate de calcium. Pour le traitement au liant aérien, la chaux utilisée est de type PROVIACAL ST mise à disposition par Lhoist dont la teneur en $\mathrm{CaO}$ est supérieure à $90 \%$ et la teneur en $\mathrm{MgO}$ est inférieure à $2 \%$.

Pour les taux de traitement considérés dans cette étude, nous avons adopté des taux combinés de traitement compris entre 0 et $6 \%$ pour assurer une viabilité économique des formulations testées et couvrir les taux utilisés habituellement dans ce domaine.

\section{2}

\section{Méthodes}

Pour la valorisation des sédiments en technique routière, la connaissance des propriétés physico-chimiques permet non seulement d'identifier la nature des sédiments marins mais aussi d'optimiser les formulations élaborées à base de liants. La quantité élevée d'eau, la proportion de matières organiques suspectée et la finesse du matériau peuvent avoir une influence néfaste sur l'effet du liant et du taux utilisé. Par conséquent, l'identification précise de ces caractéristiques sur le matériau brut est nécessaire avant d'entreprendre un quelconque traitement. Dans ce travail, ces caractéristiques sont explorées à travers la détermination de la teneur en eau initiale, la limite de liquidité, la limite de plasticité, la distribution granulométrique, la valeur au bleu de méthylène et la perte au feu du matériau. 
Après l'identification des caractéristiques physiques et le prétraitement des sédiments (infiltration, séchage, broyage, malaxage avec l'eau, préparation des matériaux à la teneur en eau optimale), le traitement aux liants est effectué de telle sorte à identifier l'effet des liants à différents dosages sur l'évolution des caractéristiques mécaniques mais également l'évolution de ces caractéristiques dans le temps. Les formulations ainsi explorées sont notées SDXL ou SDXC (avec SD : sédiment de dragage, $\mathrm{X}$ : le \% de liant, $\mathrm{L}$ : chaux, $\mathrm{C}$ : ciment). Pour chaque type de liant deux taux de traitements sont testés à savoir un taux de $3 \%$ et un taux de $6 \%$. Ce taux de traitement est évalué par rapport à la masse sèche du sédiment. Les éprouvettes cylindriques sont confectionnées basées sur les teneurs en eau optimales et les densités sèches maximales déterminées par essai Proctor Modifié définie par NF P 94-093 (1999) pour toutes les formulations. Les modalités de confection des éprouvettes sont décrites dans la norme NF EN 13286-53 (2005). L'évaluation de l'aptitude d'utilisation des sédiments en assise de chaussée est généralement mesurée par la résistance en traction et le module élastique du matériau à 360 jours. Les valeurs de ces paramètres sont calculées sur deux échantillons de même qualité. Dans cette étude, des essais de traction indirecte, de compression directe et de mesure de module sur les matériaux développés sont réalisés à différentes échéances : $14,28,60,90$ et 360 jours. Les rapports de contrainte ou de rigidité à différentes échéances sont calculés $\left(R_{t j} /\right.$ $R_{t 360 j}$ et $E_{t j} / E_{t 360 j}, j=28$ ou 90 jours) puis comparés avec ceux définis sur les matériaux standards par la norme NF P 98-113 (1994).

Les essais de compression, de traction indirecte et de mesure de modules sont effectués sur des échantillons standards avec un diamètre de $50 \mathrm{~mm}$ et une hauteur de $100 \mathrm{~mm}$. Ces derniers sont conservés pendant la période de cure dans des boîtes étanches en plastique à une température ambiante de $20^{\circ} \mathrm{C} \pm 1{ }^{\circ} \mathrm{C}$.

\section{3}

\section{Résultats}

\section{Caractérisation des sédiments}

Selon le Guide technique pour la réalisation des remblais et des couches de forme dans le secteur des travaux routiers en France, la distribution granulométrique constitue le premier paramètre d'entrée dans les tables de classification. Dans cette étude, la courbe granulométrique des sédiments est déterminée par le granulomètre laser de type COULTER LS 230 (Fig. 2). A partir de cette courbe, on peut définir le sédiment comme un matériau limoneux et argileux avec une fraction argileuse $(<2 \mu \mathrm{m})$ égale à $14,5 \%$, une fraction sableuse $(>63 \mu \mathrm{m})$ de $10,8 \%$ et, enfin, une fraction limoneuse $(63 \mu \mathrm{m}>\%>2 \mu \mathrm{m})$ de $74,7 \%$. Ces résultats mettent en évidence le caractère extrêmement fin du sédiment étudié.

En raison de la nature fine des sédiments, un paramètre important pour évaluer le comportement de ces matériaux peut être la connaissance de ses limites de consistance. Dans cette étude, la mesure de la limite de liquidité a été effectuée au travers de l'essai au cône comme définis respectivement dans la norme française

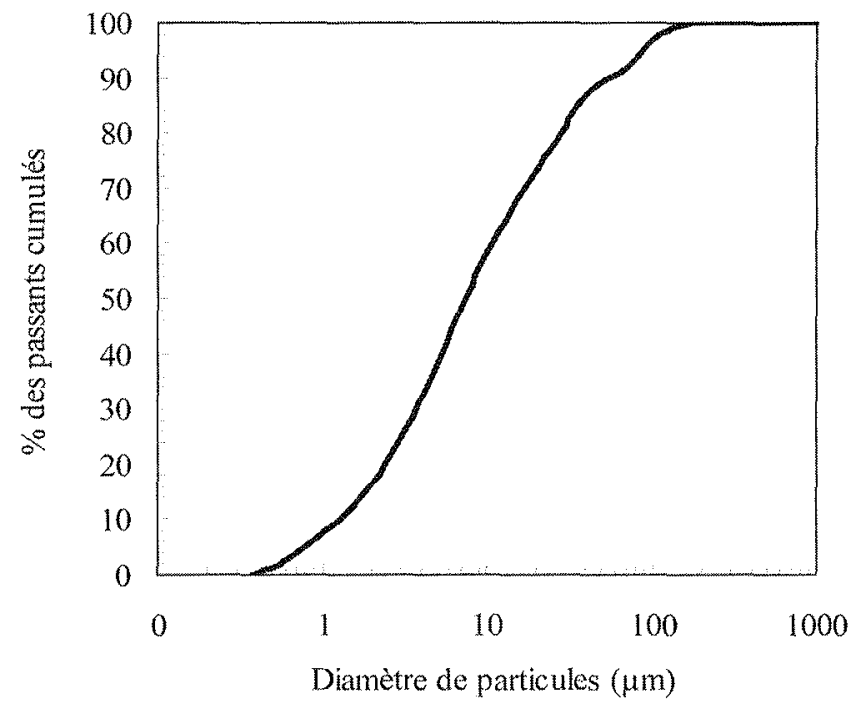

Re. 2 Distribution granulométrique des sédiments bruts.

Particle size distribution of raw sediments.

NF P 94-052-1 (1995) et la norme anglaise BS1377-2 (1990). La limite de plasticité a été approchée par l'estimation de la teneur en eau pour laquelle une pénétration de $1,7 \mathrm{~mm}$ et $2 \mathrm{~mm}$ est enregistrée respectivement en adéquation avec les travaux de Feng (2000) et Zentar et al. (2009). Sur la figure 3, la variation de la teneur en eau en fonction de la pénétration du cône est présentée. A partir des définitions ci-dessus, la teneur en eau mesurée pour un enfoncement du cône de $17 \mathrm{~mm}$ et de $20 \mathrm{~mm}$ est respectivement de $50,8 \%$ et $55,4 \%$, alors que la limite de plasticité estimée pour un enfoncement du cône de $1,7 \mathrm{~mm}$ et $2 \mathrm{~mm}$ de pénétration est respectivement de $27 \%$ et $27,5 \%$. Ces résultats conduisent à un indice de plasticité variant de $23,8 \%$ à $27,9 \%$ en fonction de la définition choisie pour définir la limite de liquidité et la limite de plasticité.

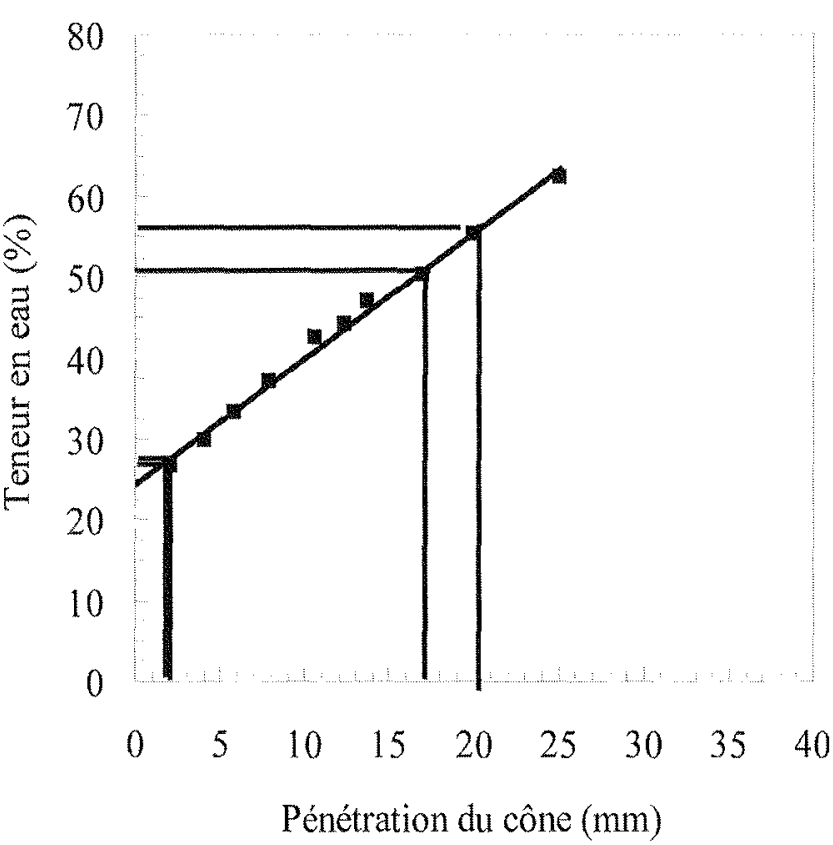

. 16 . 3 Teneur en eau en fonction de la pénétration du cône.

Water content with cone penetration. 
Sur la base de ces résultats, il apparaît que la classification du matériau sur le diagramme de plasticité de Casagrande n'est pas modifiée (Fig. 4). Les sédiments du port Est de Dunkerque sont ainsi associés à la classe de matériaux dits " très plastiques » et se regroupent autour de la ligne A qui sépare les limons des argiles.

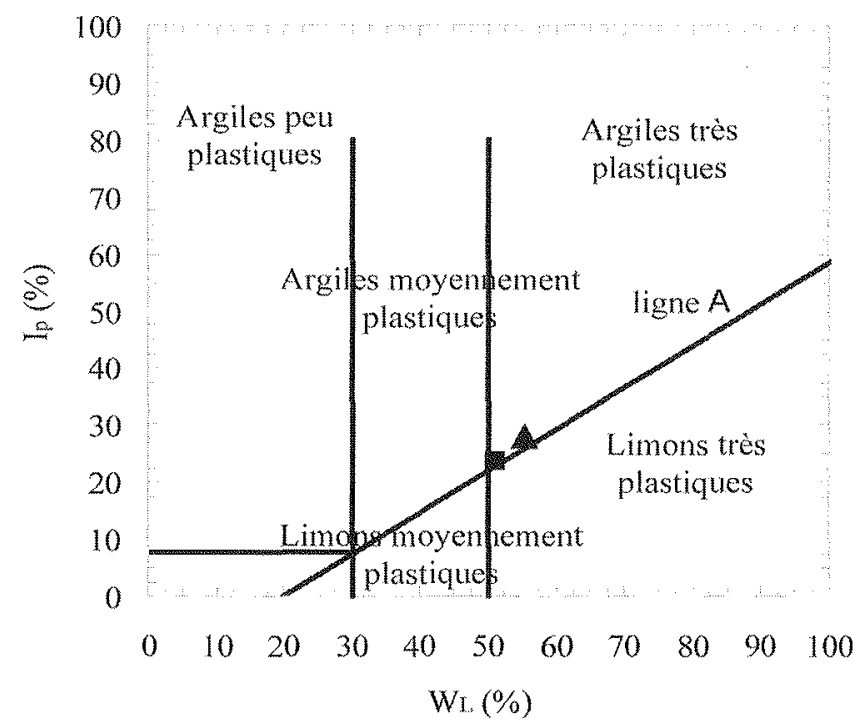

16. 4 Classification des sédiments selon le diagramme de plasticité de Casagrande. Sediment classification according to Casagrande plasticity chart.

A partir de la connaissance de la granulométrie et de l'indice de plasticité et d'après l'arbre de décision proposé par Currin et al. (1976) pour les sols, on peut conclure que selon les paramètres mesurés sur les sédiments de dragage, le traitement à la chaux ou au ciment est adéquat $\left(\mathrm{I}_{\mathrm{p}}<30\right.$ et passant à $\left.75 \mu \mathrm{m}>25 \%\right)$.

En complément des résultats relatifs à l'analyse de la courbe granulométrique et des limites de consistances du sédiment, sur le tableau I sont reportés les résultats des caractéristiques physiques du sédiment étudié ainsi que les normes d'essais utilisés pour l'évaluation de chaque paramètre. Il apparaît des résultats une teneur en eau initiale moyenne de $106,5 \%$, une teneur en matière organique de $4,4 \%$, une valeur au bleu de méthylène de 2,2 g/100 g et une masse volumique absolue de $2,58 \mathrm{~g} / \mathrm{cm}^{3}$. Les teneurs élevées en eau et en matière organiques peuvent être nuisibles au processus de solidification/stabilisation des sédiments marins. La valeur du bleue moyenne atteste de l'argilosité modérée du matériau et enfin la faible valeur de la masse volumique absolue est attribuée à la présence de matières organiques. Pour confirmer cette dernière hypothèse, la mesure de la masse volumique absolue des sédiments calcinés à $450^{\circ} \mathrm{C}$ pendant $3 \mathrm{~h}$ a été effectuée. Le résultat obtenu, de 2,70 $\mathrm{g} / \mathrm{cm}^{3}$, est comparable avec la valeur préconisée pour des matériaux standards.

\section{$x$}

\section{Évolution de la résistance en compression et en traction des sédiments traités à la chaux ou au ciment}

Afin d'évaluer l'évolution de la résistance à la compression $\left(\mathrm{R}_{\mathrm{c}}\right)$ et à la traction $\left(\mathrm{R}_{\mathrm{t}}\right)$ à long terme des sédiments traités à la chaux et au ciment, des essais de compression directe et indirecte sur des éprouvettes cylindriques de $5 \mathrm{~cm}$ de diamètre et $10 \mathrm{~cm}$ de hauteur ont été réalisés à différentes échéances s'étalant de 28 jours à 360 jours. Les résultats de ces essais sont reportés sur les figures 5 et 6 . De ces courbes, il apparaît une augmentation des résistances importante avec le type de traitement (résistance mesurée plus importante pour un traitement au ciment) mai également avec le taux de traitement.

Il apparaît également, que fonction du type de traitement la cinétique des résistances à la compression et à la traction sont différentes. Comme le montre la figure 5, la résistance à la traction à différentes échéances (de 28 jours à 360 jours) augmente pour les matériaux formulés sauf la résistance de SD6L à 360 jours. La figure 6 montre une amélioration de la résistance à la compression de 28 jours à 90 jours mais une diminution de résistance à 360 jours pour le traitement à la chaux ou au ciment. Pour les sédiments traités, l'évolution des résistances à la compression et à la traction de 28 jours à 90 jours est représentative et concordante avec les résultats obtenus par Kolias et al. (2005) et Hossain et al. (2007). Les résistances à la compression à 360 jours obtenues dans la présente étude sont souvent inférieures.

\begin{tabular}{|c|c|c|c|}
\hline \multirow[t]{3}{*}{ TABLAUI } & \multicolumn{3}{|c|}{$\begin{array}{l}\text { Caractérisation des sédiments dragués du port de Dunkerque. } \\
\text { Characterization of sediments dredged from Dunkirk Port. }\end{array}$} \\
\hline & \multirow{2}{*}{ Parametres } & \multicolumn{2}{|c|}{ serenticrits } \\
\hline & & Watersing & Whings \\
\hline & $(\%)<2 \mu \mathrm{m}$ & 14,5 & \\
\hline & $\mathrm{m}<(\%)<63 \mu \mathrm{m}$ & 74,7 & NF 13320-1 \\
\hline & $(\%)>63 \mu \mathrm{m}$ & 10,8 & \\
\hline Lim & te de liquidité (\%) & $50,8 / 55,4$ & $\begin{array}{c}\text { NF P 94-052-1 or } \\
\text { BS1377-2 }\end{array}$ \\
\hline Limi & te de plasticité (\%) & $27,0 / 27,5$ & NF P 94-051 \\
\hline Indi & e de plasticité (\%) & $23,8 / 27,9$ & / \\
\hline Tenes & r en eau initiale (\%) & 106,5 & NF P94-050 \\
\hline Valeur de bl & eu de méthylène $(\mathrm{g} / 100 \mathrm{~g})$ & 2,2 & NF P 94-068 \\
\hline Mat & ère organique (\%) & 4,4 & XР P94-047 \\
\hline Masse vo & umique absolue $\left(\mathrm{g} / \mathrm{cm}^{3}\right)$ & 2,58 & NF EN 196-6 \\
\hline
\end{tabular}




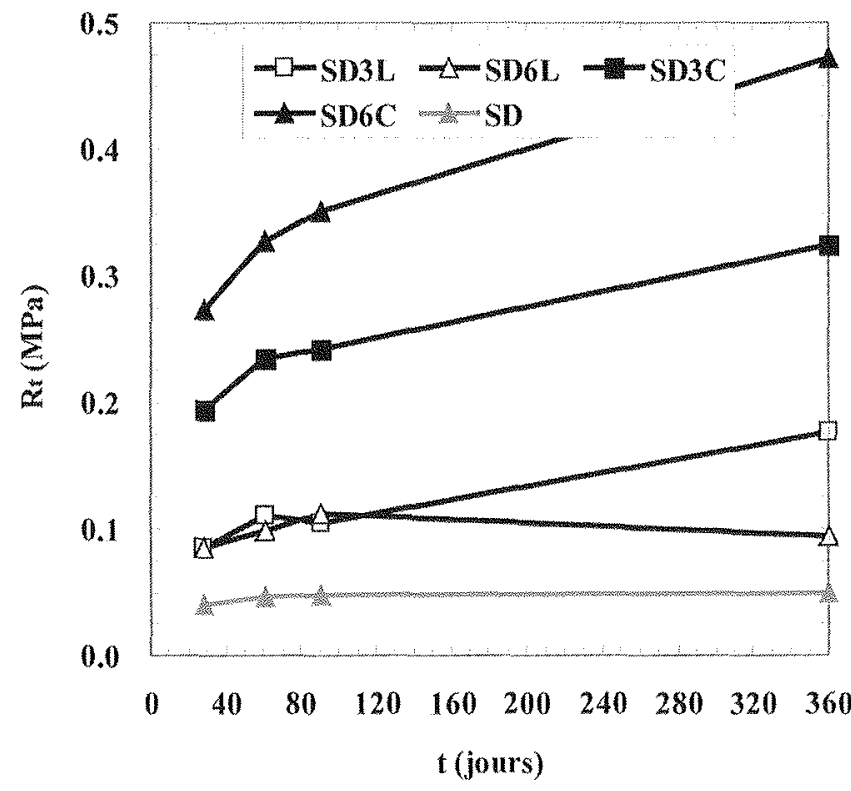

F6. $s$ Évolution de $R_{t}$ en fonction du temps $t$. Evolution of $\mathrm{R}_{\mathrm{t}}$ with time $\mathrm{t}$.

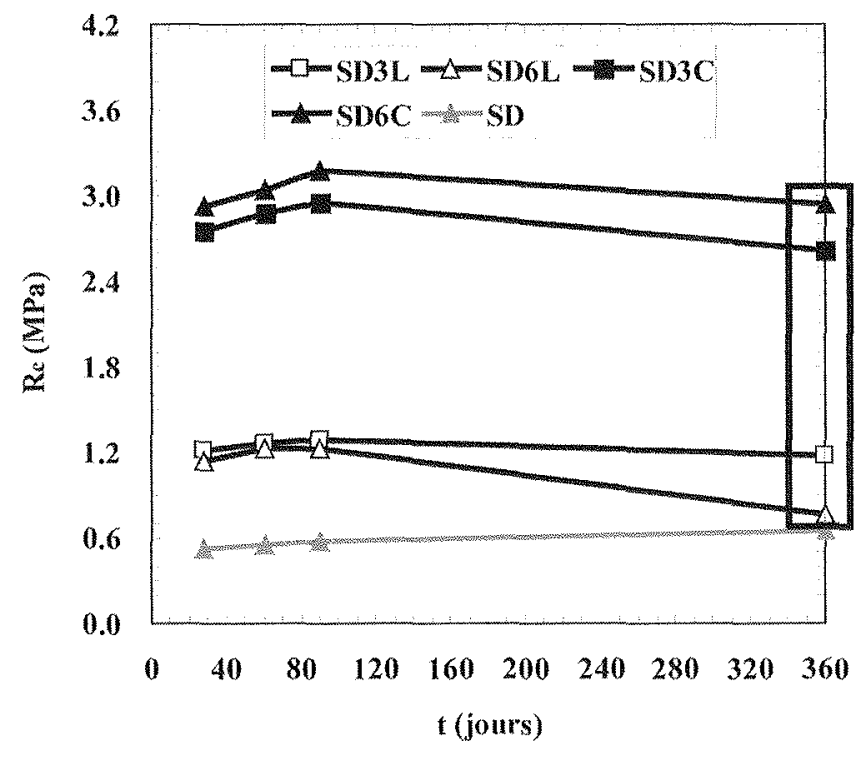

F6. 6 Évolution de $\mathrm{R}_{c}$ en fonction du temps $\mathrm{t}$. Evolution of $\mathrm{R}$ with time $\mathrm{t}$.

Pour les essais de compression simple réalisés sur les sédiments marins stabilisés à la chaux ou au ciment à 360 jours, les courbes contrainte-déformation obtenues sont reproduites sur la figure 7. A partir de ces courbes, on constate d'une part une augmentation des résistances à la rupture avec le taux et le type de traitement utilisé comme discuté ci-dessus (sauf SD6L) mais également une évolution sensible de la rigidité du matériau avec les deux paramètres précitées. Les résistances et les déformations à la rupture à 360 jours sont indiquées dans le tableau II. Le traitement avec la chaux ou le ciment induit une diminution de la déformation à la rupture de $2,61 \%$ à une valeur inférieure à $2 \%$.

Pour mieux appréhender l'évolution du taux de traitement et du type de traitement sur l'évolution de la rigidité du matériau, sur la figure 8 sont reportés les résultats d'interprétation des essais réalisés en com-



Fic 7 Relations entre contrainte-déformation des sédiments.

Stress-strain relations of sediments.

TABEEu I Résistances et déformations à rupture des sédiments à 360 jours.

Strength and deformation at rupture of sediments at 360 days.

\begin{tabular}{|c|c|c|c|}
\hline Materialus & $\mathrm{T}, \mathrm{k}, \mathrm{MP})$ & f & 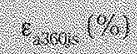 \\
\hline SD & 0,65 & 0,05 & 2,61 \\
\hline SD3L & 1,17 & 0,18 & 1,00 \\
\hline SD6L & 0,77 & 0,09 & 0,93 \\
\hline SD3C & 2,61 & 0,32 & 1,99 \\
\hline SD6C & 2,94 & 0,47 & 1,40 \\
\hline
\end{tabular}

pression et en traction sur l'évolution du module (E) évalué selon les normes NF P 98-114-3 (2001) et NF P 98-232-3 (2001). Pour les formulations SD3L et SD6L, il apparaît que le module d'élasticité est clairement amélioré par l'ajout de la chaux en fonction du temps $(t)$ de 28 jours à 360 jours. En ce qui concerne le traitement à $3 \%$ et $6 \%$ de ciment, on trouve une diminution du module d'élasticité à 360 jours par rapport à celui à 90 jours.

Pour certaines formulations, les résistances et les modules d'élasticité à 360 jours sont inférieurs à ceux mesurés à 90 jours après le traitement à la chaux ou au ciment. Cela semble en contradiction avec les travaux de Okyay et Dias (2010) (Fig. 9). Une probable explication pour ce phénomène anormal est présenté ci-dessous de point de vue de condition de stockage et de réaction chimique entre chaux/ciment et sédiments (ou impuretés dans les sédiments). Cela nous aide de clarifier les causes de ce problème anormal afin de faciliter les futures études.

Un élément d'explication de cette chute de résistance peut être l'effet de stockage des échantillons, d'inégale répartition des contraintes produit par réaction chimique entre ciment/chaux et sédiments ou de pathologie de développement très lentement au sein du matériau. Comme montré sur la figure $10 \mathrm{a}-\mathrm{c}$, une analyse fine des éprouvettes à 360 jours a révélé que certains éprouvettes ont largement gonflé d'autres font apparemment des fissurations, en particulier celles à base de ciment. Comme montré sur la figure $10 \mathrm{~b}-\mathrm{c}$, cela peut expliquer les présents résultats à 360 jours. 


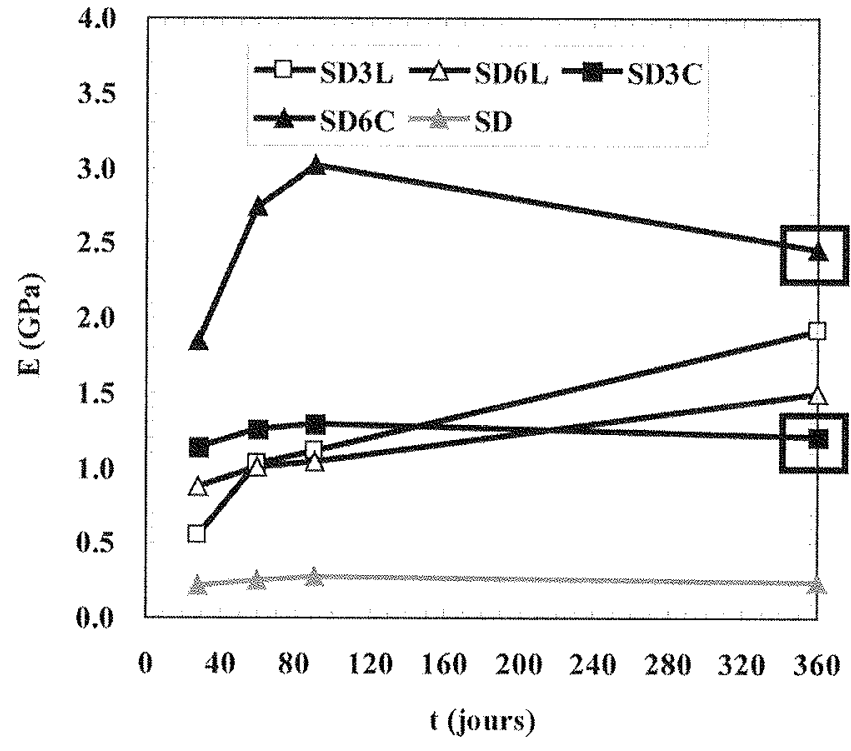

ric a Évolution de $\mathrm{E}$ en fonction du temps $\mathrm{t}$. Evolution of E with time $t$.

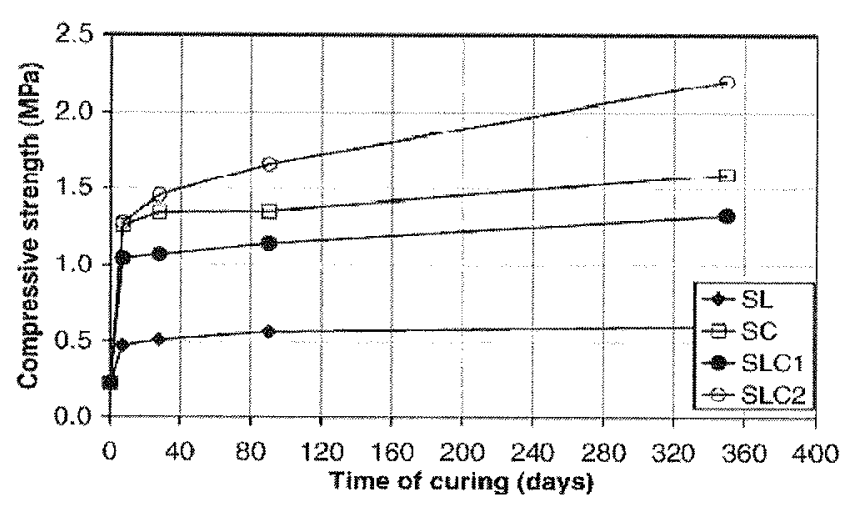

H6.9. Résultats de Okyay et Dias (2010). Results of Okyay and Dias (2010).

\section{6.}

\section{Classification des matériaux formulés}

Dans le domaine routier, la résistance en traction et le module d'élasticité sont les principaux paramètres qui permettent de déterminer l'aptitude du matériau pour une utilisation en assise de chaussée. Les résultats issus des essais réalisés dans ce travail sont reportés sur l'abaque de classification selon la norme NF P 98 114-3 (2001) et NF P 98-113 (1994).

A partir de résultats présentés sur la figure 11, il apparait que seules les formulations SD3C et SD6C appartiennent à la classe $\mathrm{S} 2$, et montrent des performances mécaniques adéquates pour une utilisation en couche d'assise de chaussée. Les autres matériaux se situent dans la zone de classe S1 et S0, ils ne peuvent pas être valorisés comme matériaux d'assise de chaussée. On observe ainsi que le traitement avec le ciment est la meilleure solution pour l'amélioration des propriétés mécaniques des sédiments marins.

A partir de résultats présentés sur la figure 12, il apparaît qu'à partir des prédictions sur la base d'essais réalisés à 28 jours pour les sédiment traités au ciment et à partir d'essais à 90 jours pour les sédiments traités à la chaux on arrive au même conclusion que sur la base d'essais réalisés à 360 jours. Autrement, les prédictions obtenues sur la base d'essais réalisés à 28 et 90 jours restent valables. Toutefois, une analyse plus détaillée des valeurs mesurées et prédites dans cette étude nous amène à discuter les rapports préconisés dans les normes NF P 98 114-3 (2001) et NF P 98-113 (1994).

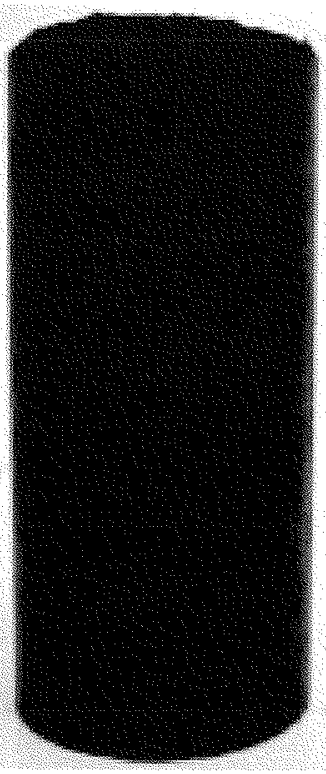
(a) Échantillon intact
Intact sample

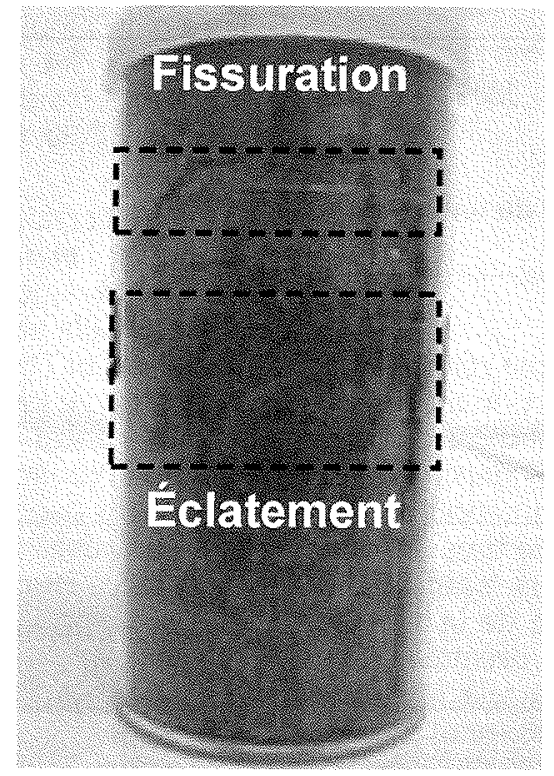

(b) Échantillon éclaté ou fissuré Broken and cracked sample

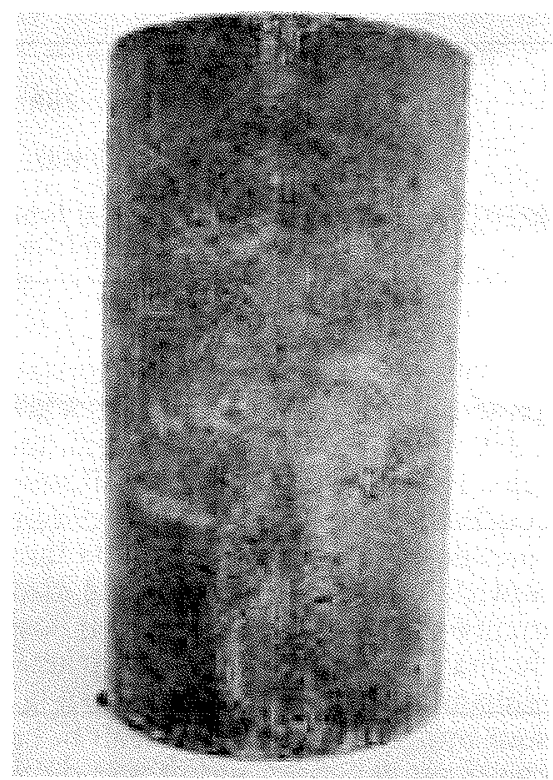

(c) Échantillon gonflé Swelled sample 


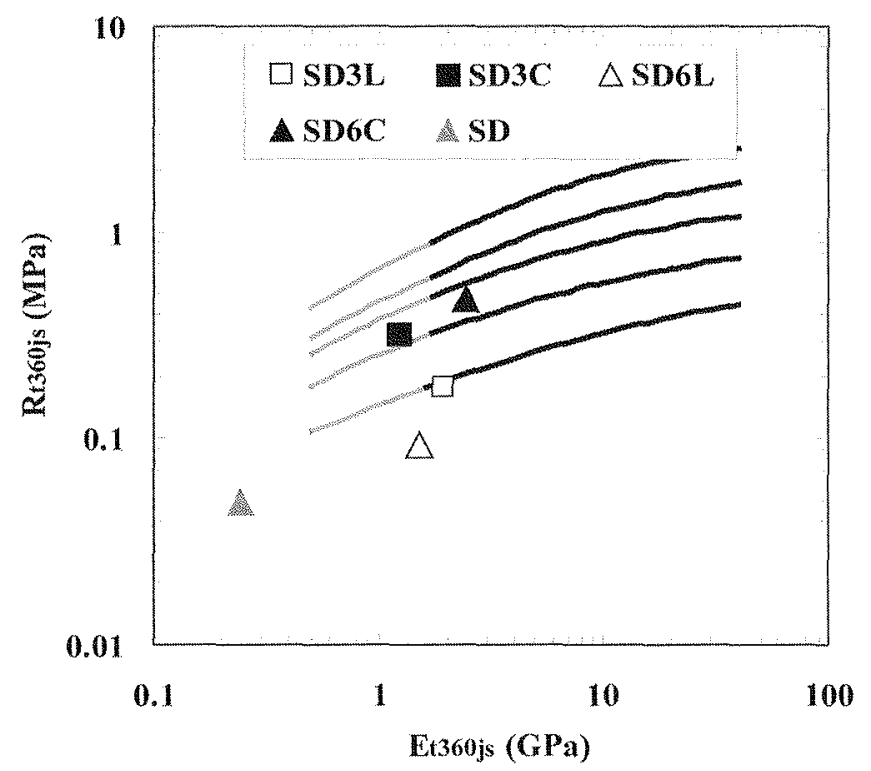

Fic. 11. Classification des matériaux sur la base de mesures effectuées à 360 jours.

Material classification based on measurements performed at 360 days.

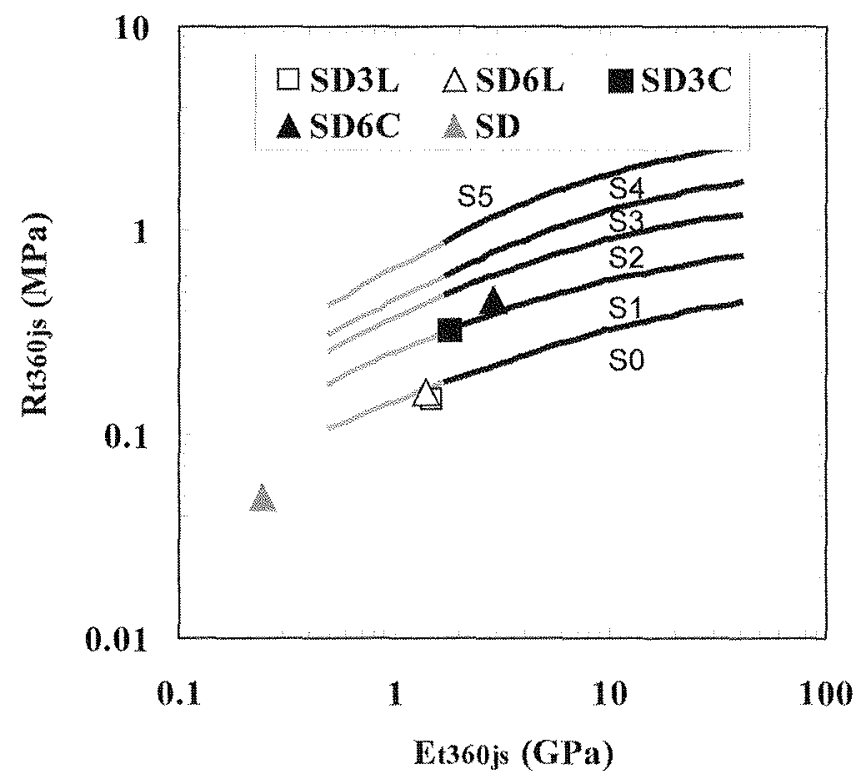

16. 12 Classification des matériaux sur la base de prédictions à partir d'essais à 28 et 90 jours.

Material classification based on predictions of test results at 28 and 90 days.

\section{$+4$}

\section{Comparaison des rapports $R_{t j} / R_{t 360 j}$ et $E_{i j} / E_{t 360 j}$}

Quand la période d'étude ne permet pas d'effectuer la mesure à 360 jours, les valeurs à 360 jours sont déduites à partir de résultats obtenus à 28 jours pour des matériaux traités au ciment et à partir de résultats obtenus après 90 jours pour des matériaux traités à la chaux en utilisant des coefficients empiriques comme décrit dans l'équation (1) et (2).

$$
\frac{R_{t 28 j s}}{R_{t 360 j s}}=0,60 \quad \frac{E_{28 j s}}{E_{360 j s}}=0,65
$$

Pour les matériaux traités au ciment

$$
\frac{R_{i 90 j s}}{R_{t 360 j s}}=0,70 \quad \frac{E_{90 j s}}{E_{360 j s}}=0,75
$$

Pour les matériaux traités à la chaux

A partir des travaux réalisés dans la présente étude, ces rapports $\left(R_{t t} / R_{t 360 j}\right.$ et $\left.E_{t j} / E_{t 360 j}\right)$ sont évalués dans les tableaux III et IV. Il apparaît des résultats, à l'exception des essais sur SD6L, que les valeurs sont légèrement en dessous des valeurs proposées pour l'estimation des résistances en traction des matériaux à 360 jours toutefois systématiquement au-dessus des valeurs proposées pour l'estimation des modules d'élasticité à 360 jours. Cet aspect contradictoire semble se compenser en partie comme montré à partir des figures 11 et 12 où le classement des matériaux au final n'est que

\begin{tabular}{|c|c|c|c|c|c|c|}
\hline treBt & \multicolumn{6}{|c|}{  } \\
\hline Marentur & MPro & Nest & 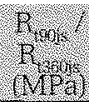 & (MFa) & (r.ypa) & 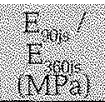 \\
\hline S & 0,10 & & 56 & 1 & 1,47 & 0 , \\
\hline SD6L & 0,11 & 0,10 & 1,38 & 1,05 & 1,10 & 0,9 \\
\hline
\end{tabular}
très peu affecté.

\begin{tabular}{|c|c|c|c|c|c|c|}
\hline TABDE & \multicolumn{6}{|c|}{ 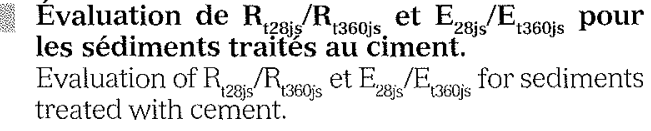 } \\
\hline Marernatu & Nos & Neris & $\begin{array}{l}p^{2} \\
n^{3}\end{array}$ &  & $\frac{\mathrm{E}_{3}}{\mathrm{M}}$ & 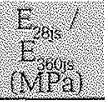 \\
\hline & 0,20 & & 0,63 & 1,14 & 1,21 & 0,94 \\
\hline SD6C & 0,27 & 0,47 & 0,58 & 1,85 & 2,46 & 0,75 \\
\hline
\end{tabular}

\section{5 \\ Rapport entre $R_{c}$ et $R_{t}$ à différentes échéances}

Pour compléter les informations relatives au rapport de contrainte entre la résistance à la compression $R_{c}$ et la résistance à la traction $R_{\text {t }}$ pour les sédiments marins, nous avons tenté d'évaluer l'évolution de ce rapport avec les différentes échéances $(28,90$ et 360 jours). Les rapports $R_{c} / R_{\text {s }}$ sont calculés en utilisant la valeur moyenne de $R_{c}$ et $R_{t}$ de deux échantillons pour chaque formulation à différentes échéances. Les résultats sont reportés sur la figure 13 avec des droites de régressions. Les rapports $R_{c} / R_{t}$ et les coefficients de corrélation $R^{2}$ mesurés sont reportés dans le tableau $V$. Les rapports $R / R_{t}$ des matériaux diminuent de 10,8 à 6 avec le temps de conservation de 28 jours à 360 jours et les coefficients de corrélation restent supérieurs à $93,5 \%$. Il semble que les rapports $R / R_{t}$ ont tendance à diminuer avec l'échéance. Mais il faut pour cela une grande quantité de résultats à long terme afin de vérifier cette conclusion. 


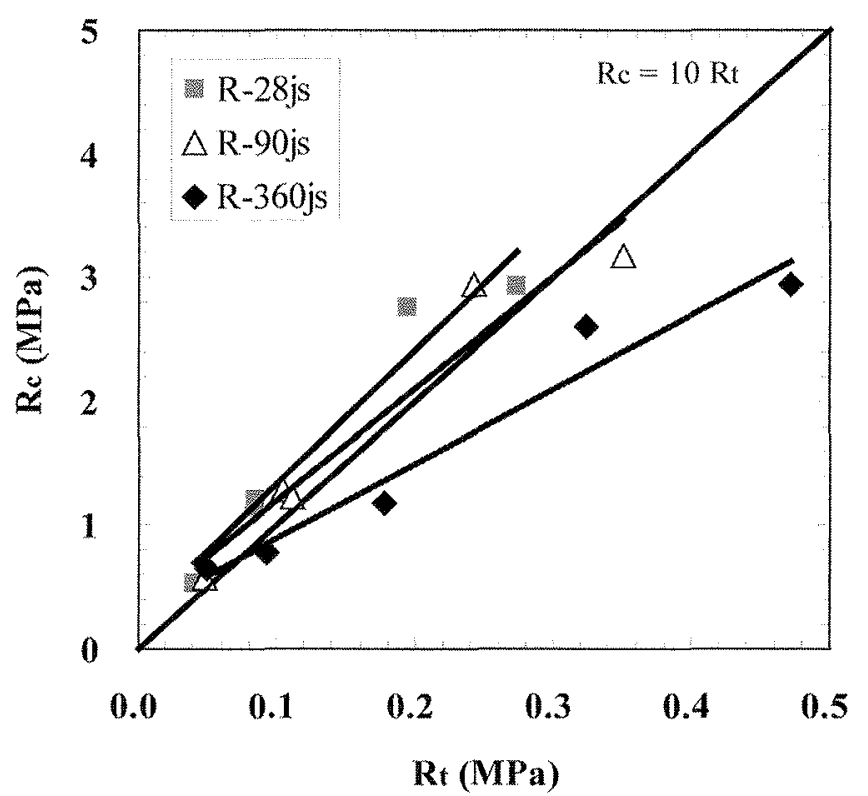

FG. 13 Rapport entre $R_{c}$ et $R_{t}$ à différentes échéances.

Relationship between $R_{c}$ and $R_{t}$ at different curing times.

\begin{tabular}{|c|c|c|c|}
\hline TABEAU V & \multicolumn{3}{|c|}{ 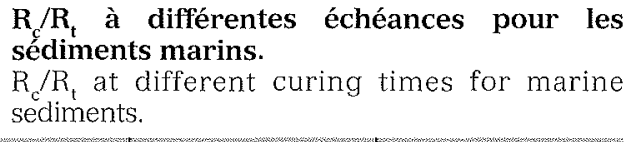 } \\
\hline \multicolumn{2}{|c|}{ ferterance $(10 \mathrm{rr})$} & R. & Ris $(\%)$ \\
\hline \multicolumn{2}{|l|}{28} & 10,8 & 94,2 \\
\hline \multicolumn{2}{|l|}{90} & 9,1 & 93,7 \\
\hline \multicolumn{2}{|l|}{360} & 6,0 & 95,2 \\
\hline
\end{tabular}

4

\section{Conclusion}

A l'issue de la phase de caractérisation de paramètres physiques, les sédiments se présentent comme des matériaux extrêmement fins, très humides et organiques. Sur la base de la granulométrie, les sédiments peuvent être identifiés comme des limons silteux et sur la base des limites de consistances ces derniers sont à la limite des argiles et des limons moyennement ou très plastiques.

Dans cette étude, la propriété mécanique à long terme est explorée sur les sédiments traités au ciment ou à la chaux en utilisant l'essai en compression et l'essai en traction. A partir des résultats des relations contrainte-déformation à 360 jours, l'ajout du ciment ou de chaux diminue la déformation à la rupture par rapport aux sédiments bruts, lorsque la résistance en compression et en traction augmente. En ce qui concerne la diminution des résistances et des modules d'élasticité à 360 jours pour certaines formulations, un léger gonflement et une faible fissuration sont observés sur les échantillons à 360 jours.

En terme de classification, les sédiments traités à $3 \%$ de ciment et $6 \%$ de ciment se situent dans la zone de classe S2, ils sont ainsi adéquats pour une utilisation en couche d'assise de chaussée. Il apparaît qu'à partir des prédictions sur la base d'essais réalisés à 28 jours pour les sédiments traités au ciment et à partir d'essais à 90 jours pour les sédiments traités à la chaux on arrive à la même conclusion en terme de classification que sur la base d'essais réalisés à 360 jours. En terme de rapports $R_{t j} / R_{t 360 j}$ et $E_{t j} / E_{t 360 j}$ une différence entre les rapports calcules et les rapports empiriques définis par les normes françaises NF P 98-113 (1994) et NF P 98 114-3 (2001) est observée. En terme de rapport $R_{c} / R_{t}$ pour les sédiments marins traités, ce rapport diminue de 10,8 à 6,0 entre 28 jours et 360 jours.

\section{RESHROENEMTS}

Les auteurs remercient le China Scholarship Council (CSC), I'École des mines de Douai et le port autonome de Dunkerque pour le financement des travaux de thèse et l'approvisionnement en matériaux. 
BS1377-2 - Method of test for soils for civil engineering purposes : classification test (BS1377-2). British Standards Institution (BSI), London, UK, 1990.

CETMEF - Enquête dragage 2008, Analyse des données. CETMEF, 2010.

Currin D.D., Allen J.J., Little D.N. - Validation of soil stabilization index system with manual development. Report FJSRLTR-76-0006, Frank J. Seiler Research Laboratory, United States Air Force Academy, 1976.

Feng T.W. - Fall-cone penetration and water content relationship of clay. Géotechnique, vol. $50, \mathrm{n}^{\circ} 2,2000$, p. 181-187.

Hamer K., Karius V. - Brick production with dredged harbour sediments, An industrial-scale experiment. Waste Manage ment, vol. 22, n 5, 2002, p. 521-530.

Hossain K.M.A., Lachemi M., Easa S. - Stabilized soils for construction applications incorporating natural resources of Papua new Guinea. Resources, Conservation and Recycling, vol. 51, n 4, 2007, p. 711-731.

Jones K.W., Feng H., Stern E.A., Lodge J., Clesceri N.L. - Dredged material decontamination demonstration for the port of New York/New Jersey. Journal of Hazardous Materials, vol. 85, $\mathrm{n}^{\circ}$ 1, 2001, p. 127-143.

Kolias S., Kasselouri-Rigopoulou V., Karahalios A. - Stabilisation of clayey soils with high calcium fly ash and cement. Cement \& Concrete Composites, vol. 27 $\mathrm{n}^{\circ} 2,2005$, p. 301-313.

Levacher D., Colin D., Perroni A.C., Duan Z., Sun L. - Recyclage et valorisation de sédiments fins de dragage à usage de matériaux routiers. IX ${ }^{\text {es }}$ Journées nationales Génie civil-Génie côtier, Brest, France, 12-14 septembre 2006, p. 603-612

NF EN 196-6 - Methods of testing cement Determination of fineness. 1990.

NF ISO13320-1 - Analyse granulométriqueMéthodes par diffraction laser. Partie 1 : principes généraux. Association fran caise de normalisation, 2000.

NF P94-050 - Sols : Détermination de la teneur en eau pondérale des matériaux. Association française de normalisation, 1995.
NF P 94-051 - Sols : reconnaissance et essais - Détermination des limites d'Atterberg-Limite de liquidité à la coupelle - Limite de plasticité au rouleau. Association française de normalisation, 1993. NF P 94-052-1 - Sols : reconnaissance et essais - Détermination des limites d'Atterberg. Partie 1 : Limite de liquidité Méthode du cône de pénétration. Association française de normalisation, 1995.

NF P 94-068 - Sols : Reconnaissance et essais - Mesure de la capacité d'adsorption de bleu de méthylène d'un sol ou d'un matériau rocheux - Détermination de la valeur de bleu de méthylène d'un sol ou d'un matériau rocheux par l'essai à la tache. Association française de normalisation, 1998.

NF P 94-093 - Sols : Reconnaissance et essais - Détermination des références $d u$ compactage d'un matériau - Essai Proctor normal - Essai Proctor modifié. Association française de normalisation, 1999.

NF P 98-113 - Assises de chaussée : Sables traités aux liants hydrauliques et pouzzolaniques : Définition - Composition Classification. Association française de normalisation, 1994.

NF P 98-114-3 - Assises de chaussées Méthodologie d'étude en laboratoire des matériaux traités aux liants hydrauliques. Partie 3 : Sols traités aux liants hydrauliques éventuellement associés à la chaux. Association française de normalisation, 2001.

NF EN 13286-53 - Mélanges traités et mélanges non traités aux liants hydrauliques. Partie 53 : Méthode de confection par compression axiale des éprouvettes de matériaux traités aux liants hydrauliques. Association française de normalisation, 2005

NF P 98-232-3 - Essais relatifs aux chaussées - Détermination des caractéristiques mécaniques des matériaux traités aux liants hydrauliques. Partie 3 : Essai de compression diamétrale sur les matériaux traités aux liants hydrauliques et pouzzolaniques. Association française de normalisation, 2001.
Okyay U.S., Dias D. - Use of lime and cement treated soils as pile supported load transfer platform. Engineering Geology, vol. 114, n' 1-2, 2010, p. 34-44.

Sannier L., Levacher D., Jourdan M. Approche économique et validation de méthodes de traitements aux liants hydrauliques de sédiments marins contaminés. Revue Paralia, vol. 2, $\mathrm{n}^{\circ} \mathrm{s} 2$, 2009, p. 1-15.

Scordia P.Y., Lafhaj Z., Skoczylas F., Mongeois F - Caractérisation et valorisation en technique routière de sédiments fluviaux pollués et traités. Revue européenne de genie civil, vol. 12, n 9-10, 2008, p. 1087-1104

Silitonga E., Levacher D., Mezazigh S. Effects of the use of fly ash as a binder on the mechanical behaviour of trea ted dredged sediments. Environmental Technology, vol. $30, \mathrm{n}^{\circ} 8,2009$, p. 799 807.

Tran N.T. - Valorisation de sédiments marins et fluviaux en technique routière. Thèse de doctorat, École des mines de Douai, 2009.

Wang D.X., Abriak N.E., Zentar R., Xu W.Y. - Solidification/stabilization of dredged marine sediments for road construction. Environmental Technology, vol. $33, \mathrm{n}^{\circ} 1$, 2012, p. 95-101.

XP P94-047 - Sols : reconnaissance et essais - Détermination de la teneur pondérale en matières organiques d'un matériau - Méthode par calcination. Association française de normalisation, 1998.

Zentar R., Abriak N.E., Dubois V. - Effects of salts and organic matter on Atterberg limits of dredged marine sediments. Applied Clay Science, vol. 42, $\mathrm{n}^{\circ} 3-4$, 2009, p. 391-397.

Zentar R., Dubois V., Abriak N.E. - Mechanical behaviour and environmental impacts of a test road built with marine dredged sediments. Resources, Conservation and Recycling, vol. 52, $\mathrm{n}^{\circ} 6,2008$, p. 947-954.

Zhu Z.D., Liu S.Y. - Utilization of a new soil stabilizer for silt subgrade. Engineering Geology, vol. 97, n³-4, 2008, p. 192-198. 